\title{
Parasite load and its association with disease outcome in cutaneous leishmaniasis in Sri Lanka
}

FH Riyal ${ }^{1}$, TN Samaranayake ${ }^{1}$, J Ganeshalingam $^{2}$, AAH Priyani $^{2}$, D Munidasa $^{3}$, ND Karunaweera ${ }^{1}$

\section{Introduction}

Cutaneous leishmaniasis is an endemic vector borne disease in Sri Lanka caused by the protozoan parasite Leishmania donovani. Patients show diversity in the response to treatment which is likely influenced by multiple factors. Our previous analyses have shown that epidemiological (gender, and age) and clinical features (lesion type, size, and location) or duration of the lesion before initiating treatment do not influence the outcome. The aim of this study was to investigate the association between the parasite load as identified by histology and its association with response to therapeutic interventions.

\section{Methods}

116 patients with suspected lesions for cutaneous leishmaniasis attending the Dermatology Clinic at Teaching Hospital, Anuradhapura were recruited to the study. A $3 \mathrm{~mm}$ punch biopsy was taken from the lesion for histological analysis. An impression smear was made using the biopsy to perform microscopical investigations. Patients were followed up until the completion of treatment. Data was analysed using SPSS software.

\section{Results}

103 of the 116 patients were parasitologically confirmed as having leishmaniasis. 91 patients (mean age; $42 \pm 2.764$ years), consisting of 66 males (73\%) and 25 females (27\%) who completed treatment were included in this study. Commonest lesion type was nodules; 42 (46\%) followed by ulcers; 33 (36\%). Patients were categorized as those with a low (0 parasites); 60 (66\%), mid (1100 parasites); 8 (9\%) or high (>100 parasites); 23 (25\%) parasite load according to a modified Ridley's classification. The number of intra-lesional Sodium Stibo Gluconate (SSG) doses required until complete healing ranged from 7 to 21 doses with an average of 12 doses. (37 (41\%) of the patients healed within 11-13 doses).

The parasite load showed a positive correlation with the number of SSG doses required for complete healing $(\mathrm{r}=0.55, \mathrm{p}<0.01)$. The type or size of the lesion was not associated with the parasite load ( $\mathrm{p}>0.05)$.

\section{Conclusions}

The number of parasites in the lesion can be considered as a biological marker of response to treatment while the clinical presentation appears to be independent of the parasite load.

Keywords: Cutaneous leishmaniasis, parasite load, treatment response, Leishmania donovani

Funding: University of Colombo, Collaborative Research grant (AP/3/2/2016/CG/24) and NIAID, NIH (National Institutes of Health) grants (R01AI099602 and U01AI136033)

\footnotetext{
${ }^{1}$ Department of Parasitology, Faculty of Medicine, University of Colombo, Sri Lanka

${ }^{2}$ Department of Pathology, Faculty of Medicine, University of Colombo, Sri Lanka

${ }^{3}$ Teaching Hospital, Anuradhapura

Address for correspondence: Hasna Riyal.Telephone:+94717703026 Email: hasna.riyal@gmail.com

(iD) https://orcid.org/0000-0002-7395-9103
} 